\title{
Alterations in the long non-coding RNA transcriptome in mesangial cells treated with aldosterone in vitro
}

\author{
GAOTING QU ${ }^{1 *}$, HUIMIN SHI $^{1 *}$, BIN WANG $^{2}$, SHANWEN LI $^{1}$, AIQING ZHANG $^{1}$ and WEIHUA GAN ${ }^{1}$ \\ ${ }^{1}$ Department of Pediatric Nephrology, The Second Affiliated Hospital of Nanjing Medical University, Nanjing, Jiangsu 210003; \\ ${ }^{2}$ Division of Nephrology, Huashan Hospital and Institute of Nephrology, Fudan University, Shanghai 200040, P.R. China
}

Received November 3, 2016; Accepted June 23, 2017

DOI: $10.3892 / \mathrm{mmr} .2017 .7313$

\begin{abstract}
Clinical and experimental reports indicate that aldosterone (ALD) contributes to the progression of renal failure independent of its hemodynamic effects. However, the mechanisms remain to be completely elucidated. The aim of the present study was to investigate the alterations of long non-coding RNA (lncRNA) in mesangial cells (MCs) treated with ALD. The present study used MCs treated with $10^{-6}$ M ALD as experimental cells. Microarray techniques performed by Agilent Technologies were used to identify the profiles of differentially expressed lncRNAs between the ALD group and the control group. Pathway and gene ontology analysis were applied to determine the roles of the differentially expressed lncRNAs. Reverse transcription quantitative polymerase chain reaction (RT-qPCR) was used to quantify the differentially expressed lncRNAs. A total of 8,459 IncRNA and 13,214 mRNAs with differential expression between MCs treated with and without ALD were identified. The expression of IncRNAs was confirmed by RT-qPCR and the results were consistent with the lncRNA array. The biological functions of lncRNAs are associated with responding to external stimuli, positive regulation of biological and apoptotic processes, cell division, mitosis and nuclear division. The pathways include cell cycle and peroxisome proliferator-activated receptor signaling pathways. The present study revealed distinct sets of lncRNA expressed in MCs treated with ALD, suggesting that this class of transcripts may be involved in the pathogenesis of chronic kidney diseases.
\end{abstract}

Correspondence to: Dr Aiqing Zhang or Professor Weihua Gan, Department of Pediatric Nephrology, The Second Affiliated Hospital of Nanjing Medical University, 262 Zhongshan North Road, Nanjing, Jiangsu 210003, P.R. China

E-mail: njaiqing@njmu.edu.cn

E-mail: weihuagan@njmu.edu.cn

*Contributed equally

Key words: long non-coding RNA, aldosterone, chronic kidney disease, mesangial cells

\section{Introduction}

Chronic kidney disease (CKD) has been increasingly recognized as a major public health problem in the world (1). Therefore, improvement in the current knowledge of molecular alterations associated with CKD is required to investigate novel strategies of diagnostics and treatment of this disease. The progression of CKD is characterized by glomerular hypertrophy, mesangial cell (MC) proliferation, extracellular matrix (ECM) accumulation, glomerulosclerosis and ultimately end-stage kidney disease (2). The ECM produced by damaged MCs is a major factor in mesangial proliferation and this is observed in light-chain-related glomerular disease which is associated with an increased synthesis of tenascin by MCs (3). MC proliferation is reported in humans and in experimental animal models with chronic nephron loss, and precedes the development of secondary focal glomerulosclerosis (4-6). In addition, progressive glomerulosclerosis is a common route for the development of end-stage renal failure of any etiology. Therefore MCs serve a critical role in the maintenance of renal function, supporting the glomerular capillaries and regulating their blood flow (7). Various growth factors and cytokines, produced by the infiltrating cells during the disease process and by the local kidney cells, have been implicated in the fibrotic process (8). Among these, aldosterone (ALD) produced by the adrenal cortex and the MCs, in addition to other extra-adrenal tissues, including cardiac myocytes and vascular smooth muscle cells, serves a significant role in the pathogenesis of mesangial matrix expansion (9-19). Part of the intracellular mechanisms involved in the proliferative and fibrotic effect of ALD in CKD has been reported. For example, ALD upregulates protein synthesis, and mRNA expression of fibronectin and transforming growth factor- $\beta 1$ (TGF- $\beta 1$ ) in cultured rat MCs partly by enhancing extracellular signal-regulated kinase 1/2 (ERK1/2) and c-Jun N-terminal kinase (JNK) activities, and subsequent activity of transcription factor AP-1 (AP-1) (20,21). ALD stimulates intracellular adhesion molecule-1 and connective tissue growth factor transcription via activation of serine/threonine-protein kinase Sgk1 and nuclear transcription factor p65, which may be involved in the progression of ALD-induced mesangial fibrosis and inflammation (22). ALD stimulates the mitogen-activated protein kinase pathway, which promotes the proliferation of MCs (23). ALD can also increase plasminogen activator inhibitor-1 
mRNA and protein expression in cultured MCs (24). Although these signaling pathways have been identified to serve important roles during the pathogenesis of CKD, there remain few effective therapeutic treatments to cure CKD by targeting these molecules. Therefore, it is necessary for novel molecular potential targets to be investigated.

Long non-coding RNA (lncRNA) has received attention in the investigation of the complex mechanisms underlying malignant processes, including tumorigenesis, drug-resistance and metastasis of different types of cancer (25). In the beginning, the majority of transcriptional outputs of the mammalian genome were confirmed to be protein noncoding genes (26) and the lncRNAs were identified as transcriptional 'noise' or cloning artifacts (27). During the past decade, multiple lncRNAs have been demonstrated and confirmed to be involved in the regulation of gene transcription, chromatin methylation, post-transcriptional modification and other biological progresses (28). However, the systematic analysis of aberrant expression profiles of lncRNA in MCs treated with ALD remains to be performed. In the present study, the expression pattern of lncRNAs was investigated by high-throughput microarray in MCs treated with ALD. Furthermore, to couple the observed differential expression of lncRNA with the expression of mRNAs, an mRNA transcriptome analysis by microarray was conducted. The aim of the present study was to clarify the roles of differentially expressed lncRNAs in MCs treated with ALD and provide a novel insight into CKD pathogenesis, and to identify potential biomarkers and therapeutic targets for CKD.

\section{Materials and methods}

Cell culture. Cultured rat MCs were purchased from the China Center for Type Culture Collection (Wuhan, China). MCs from passages 7-9 were used in the experiments. The cells were maintained in Dulbecco's modified Eagle's medium (DMEM; Hyclone; GE Healthcare Life Sciences, Logan, UT, USA) supplemented with $10 \%$ fetal calf serum (FCS; Gibco; Thermo Fisher Scientific, Inc., Waltham, MA, USA) in a humid atmosphere containing $5 \% \mathrm{CO}_{2}$ at $37^{\circ} \mathrm{C}$. MCs were grown to $75 \%$ confluence in $100 \mathrm{~cm}^{2}$ flasks (BD Biosciences, Franklin Lakes, NJ, USA) were incubated in serum-free medium for $24 \mathrm{~h}$ and then treated with ALD (R\&D Systems, Inc., Minneapolis, MN, USA) at a concentration of $10^{-6} \mathrm{M}$ in a 96-well plate for $24 \mathrm{~h}$ at $37^{\circ} \mathrm{C}$.

Preparation of model. Performed as previously described by Zhang et al (29), with $10^{-6}$ M ALD-treated MCs being used as the experimental cells. An equal amount of solvent (DMEM+10\% FCS) was added to the solvent control group. Cells were cultured in 6-well plates for $24 \mathrm{~h}$. MCs were washed with PBS three times, then $200 \mu$ l EDTA (Gibco; Thermo Fisher Scientific, Inc.) was added to each well followed by $750 \mu \mathrm{TRIzol}^{\circledR}$ reagent (Invitrogen; Thermo Fisher Scientific, Inc.). The cells were stored at $-80^{\circ} \mathrm{C}$ and sent to the LncRNA Expression Microarray (Arraystar, Rockville, MD, USA).

IncRNA microarray analysis. The IncRNAs were constructed using public transcriptome databases (Refseq, www.ncbi. nlm.nih.gov/refseq; University of California Santa Cruz knowngenes, genome.ucsc.edu; and Gencode, www. gencodegenes.org), in addition to a publication (30). The lncRNA expression microarray used in the present study classifies its probes as the following subtypes: i) Enhancer lncRNAs: Contains profiling data of all lncRNAs with enhancer-like function; ii) Rinn lncRNAs: Contains profiling data of all lncRNAs based on studies by Khalil et al (31) and Guttman et al (32); iii) homeobox protein (HOX) cluster: Contains profiling data of all probes in the four HOX loci, targeting 407 discrete transcribed regions, lncRNAs and coding transcripts; iv) lncRNAs located near coding genes: These contain differentially expressed lncRNAs and nearby coding gene pairs (distance, $300 \mathrm{~kb}$ ); and v) enhancer lncRNAs located near coding genes: These contain differentially expressed enhancer-like lncRNAs and their nearby coding genes (distance, $300 \mathrm{~kb}$ ). Following hybridization and washing, processed slides were scanned with an Agilent DNA Microarray Scanner (part no. G2505B; Agilent Technologies, Inc., Santa Clara, CA, USA). Agilent Feature Extraction software version 10.7.3.1 (Agilent Technologies, Inc.) was used to analyze all acquired array images. Quantile normalization and subsequent data processing were performed using the GeneSpring GX version 11.5.1 software package (Agilent Technologies, Inc.) (33). The profile of microarray data of the 8,459 IncRNAs was detected by third-generation lncRNA microarray. The general characteristics of the differentially-expressed IncRNAs were summarized, including chromosomal, source, relationship and fold-change distribution using the most widely-used public transcriptome databases (Ensembl, www.ensembl.org/index.html; UCSC, genome.ucsc.edu/index.html; NONCODE, www.noncode.org; NCBI, www.ncbi.nlm.nih.gov). The source of the lncRNA was collected from RefSeq_NR (RefSeq validated non-coding RNA), RefSeq_XR (RefSeq un-validated non-coding RNA), mouse_ortholog (rat lncRNAs which are obtained by sequence comparison with mouse lncRNAs), 'ultra-conserved regions' among human, mouse and rat (users.soe.ucsc.edu/ jill/ultra. html), and misc_lncRNA (other sources).

Reverse transcription-quantitative polymerase chain reaction (RT-qPCR). RT-qPCR was used to verify the differential expression of seven lncRNAs and six associated genes that were detected by the IncRNA and mRNA expression microarray. The cDNA was synthesized using reverse transcriptase (Takara Bio, Inc., Otsu, Japan) and oligo (dTs) primers with $1 \mu 1$ RNA from the same samples as those used in the microarray. The reaction consisted of $2 \mu \mathrm{l} 5 \mathrm{X}$ PrimeScript buffer, $0.5 \mu \mathrm{l}$ PrimeScript RT Enzyme Mix I, $0.5 \mu \mathrm{l}$ Oligo dT Primer, $0.5 \mu \mathrm{l}$ Random 6 mers, 500 ng total RNA and RNase free $\mathrm{dH}_{2} \mathrm{O}$ up to $10 \mu \mathrm{l}$, and was performed for $15 \mathrm{~min}$ at $37^{\circ} \mathrm{C}$. Primers for each IncRNA and mRNA are reported in Table I. qPCR was performed on an Applied Biosystems ViiA ${ }^{\mathrm{TM}} 7$ Dx (Thermo Fisher Scientific, Inc.) using the SYBR-Green method, according to the manufacturer's protocol. Each RT-qPCR reaction (in $10 \mu \mathrm{l}$ ) contained $5 \mu \mathrm{l}$ SYBR-Green real-time PCR Master mix (Thermo Fisher Scientific, Inc.), $1.0 \mathrm{mM}$ primer and $1 \mu \mathrm{l}$ template cDNA. The cycling conditions consisted of an initial single cycle of $2 \mathrm{~min}$ at $50^{\circ} \mathrm{C} ; 2 \mathrm{~min}$ at $95^{\circ} \mathrm{C}$; followed by 40 cycles of $15 \mathrm{sec}$ at $95^{\circ} \mathrm{C}, 15 \mathrm{sec}$ at $56^{\circ} \mathrm{C}$ and $60 \mathrm{sec}$ at $72^{\circ} \mathrm{C}$. PCR amplifications were performed in triplicate for 
Table I. Primers for quantitative polymerase chain reaction of long non-coding RNA and mRNAs.

Primers $\left(5^{\prime}-3^{\prime}\right)$

\begin{tabular}{lll}
\cline { 2 - 3 } Genes & \multicolumn{1}{c}{ Forward } & \multicolumn{1}{c}{ Reverse } \\
\hline BC168211 & CACCTGGCCACTGTTTCCTAA & TGATACTCGGCTAGGGAAGCA \\
BC088254 & CCCAGAAAGCTCTCAGGTCCTA & TGCTGGGTGCTTTATTTACACAA \\
AF336872 & TGGCCAGGAGTGCCATTC & CCCCCCAATGCCATGA \\
AY325162 & CCCATGTCCCTCATTCATTACC & GGTGACACGAAGCATCCAAGT \\
BC168687 & CATTGCTCCTGTCTTAGGTCGTT & GGTGGCGATAGGGTTAATTCC \\
AF230638 & TCCTTTTGCAAGAATCCATACTCA & CGGTGCTAACGGTGAATCAGA \\
BC167085 & TGGAGGCCGCCAAGTGT & GAATCCCACCGGGTCACA \\
NM_001108598 & CACCTGGCCACTGTTTCCTAA & TGATACTCGGCTAGGGAAGCA \\
NM_001109190 & CCAGGCTATGAACGGTTTCC & AGTAGGGTCTGTTTGCATCCTTAGG \\
NM_001101018 & TCACCAAGACCCAGTTCAGTTAGA & GAAGGCCGTGCCAATGAG \\
NM_019347 & ACACACCTGTTGGCACTTGTCT & CGGTGGCACACCAACCA \\
NM_177962 & CCTTGCACCTGTTTCAAATCAA & GGGCAGAGGGAACGAATCA \\
NM_001108823 & AACCCTCAGGAGCCATGCT & TGGGCACTGCAGGTGAGA \\
\hline
\end{tabular}

each sample. Gene expression levels were quantified relative to the expression of GAPDH (primers: Sense, 5'-CAAGTT CAACGGCACAGTCAA-3'; antisense, 5'-TGGTGAAGA CGCCAGTAGACTC-3') using an optimized comparative $2^{-\Delta \Delta C q}$ method (34). RT-qPCR was performed in triplicate on the diluted cDNA and the experiments were performed twice in the control and ALD-treated MC groups.

Gene ontology $(G O)$ analysis and pathway analysis. Previous studies have demonstrated that IncRNAs are preferentially located next to genes with developmental functions (26). For each lncRNA locus the nearest protein-coding neighbor within $100 \mathrm{~kb}$ was identified. For antisense overlapping and intronic overlapping lncRNAs, the overlapping gene was identified. GO (www.geneontology.org) and Kyoto Encyclopedia of Genes and Genomes (KEGG) pathway analyses (www. genome.jp/kegg) were applied to determine the roles of these closest coding genes in GO terms or biological pathways.

$\mathrm{GO}$ analysis was used to assess the main function of the closest coding genes according to the GO database, which provides the key functional classifications for the National Center for Biotechnology Information (35). The ontology covers two domains: Biological processes and molecular function. Fisher's exact test is used to identify if there is more overlap between the differentially expressed list and the GO annotation list than would be expected by chance. The P-value denotes the significance of GO term enrichment in the differentially expressed genes. Pathway analysis is a functional analysis mapping genes to the KEGG pathways. The P-value (expression analysis systematic explorer-score, Fisher-P-value or Hypergeometric-P-value) denotes the significance of the pathway correlated with the conditions. $\mathrm{P}<0.05$ was considered to indicate a statistically significant difference.

Statistical analysis. Each qPCR experiment was performed at least three times. Numerical data were presented as the mean \pm standard error of the mean. Relative expression levels of IncRNAs between the two groups were analyzed using the Student's t-test. All statistical analyses were performed using SPSS software (version 18; SPSS, Inc., Chicago, IL, USA). $\mathrm{P}<0.05$ was considered to indicate a statistically significant difference.

\section{Results}

Profile of IncRNA microarray data. A gene chip study was performed in the normal and ALD-treated MC group to investigate the possible lncRNA alteration in expression using the Arraystar probe dataset, which included 8,459 lncRNAs. The IncRNAs were constructed using public transcriptome databases (Refseq, University of California Santa Cruz knowngenes and Gencode), in addition to a publication (30). The scatterplot is useful for assessing the variation in the expression of IncRNAs and coding transcripts between the two MCs (Fig. 1A); the dot above the green line represents a difference of $>2$ times. The number of points above the top and below the bottom green lines indicated lncRNAs that exhibit $>2.0$ fold-change when comparing the control and ALD-treated MC groups. By setting a filter of fold-change $>3.0$, raw $>100$ of the expression level between the ALD-treated group and control group, 5 upregulated and 2 downregulated lncRNAs were identified (Fig. 1B).

Differently expressed $m R N A s$ in the ALD-treated rat $M C$ group. An Affymetrics gene array containing 13,214 gene transcripts was used to perform a comprehensive analysis of mRNA expression in control compared with ALD-treated rat MCs group. Comparing the rat MCs treated with ALD with the control group, 180 genes were differentially expressed (fold-change $>3.0$; raw $>100$ ), of which 36 genes were upregulated and 144 genes were downregulated (Fig. 1C). A scatter-plot illustrating the expression patterns of these differentially expressed mRNAs between control and ALD-treated rat MCs is exhibited in Fig. 1D. 
A
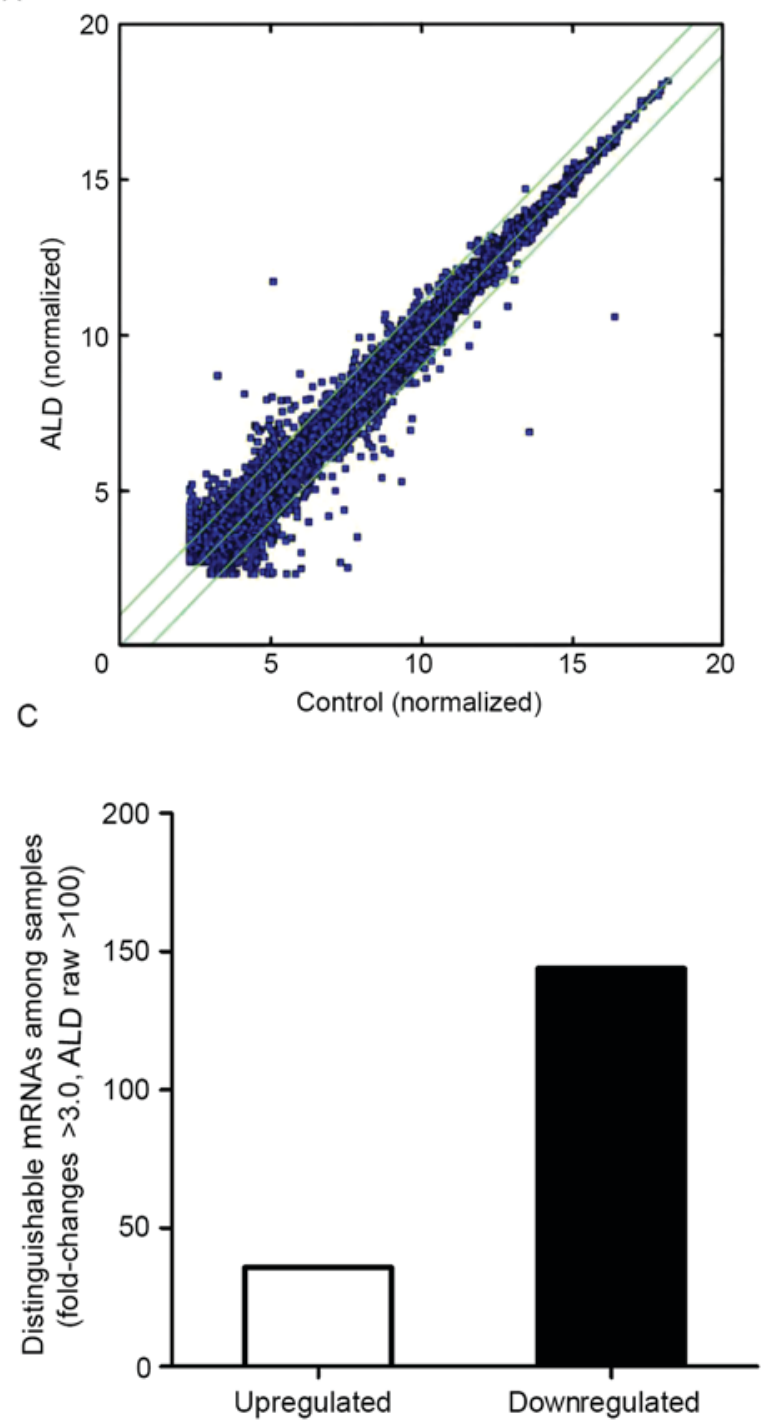

B

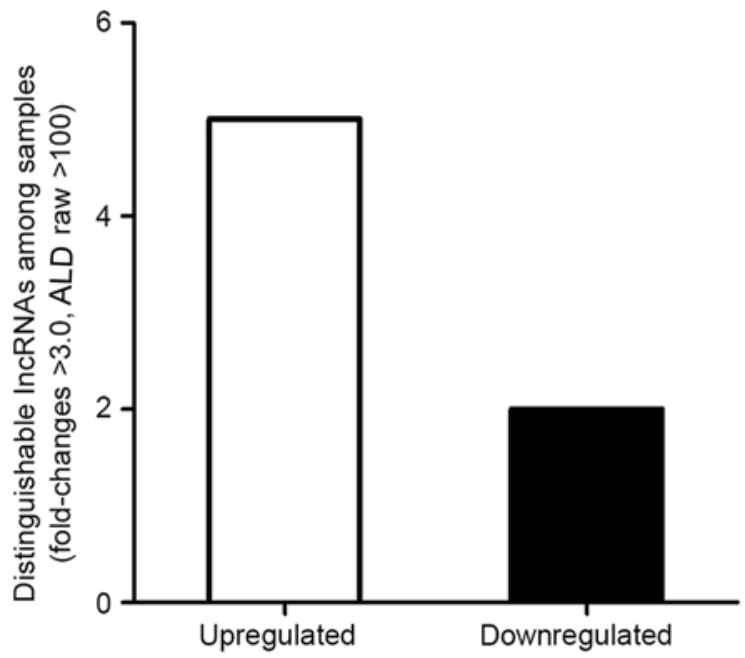

D

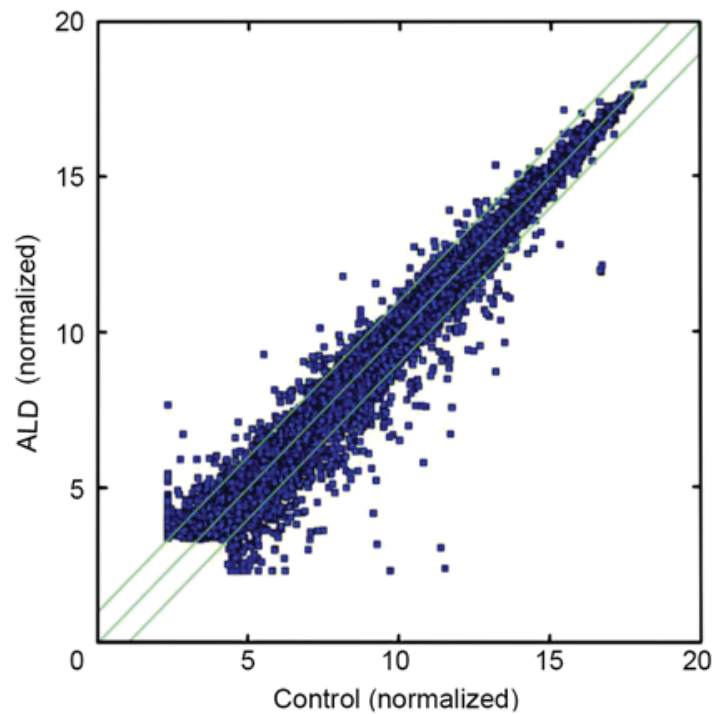

Figure 1. Scatter-plots illustrating the distinct expression patterns of differentially expressed RNAs between control and MCs treated with ALD. (A) Fold-change alterations for lncRNAs expressed in the control and ALD-treated MC group. The number of points above the top and below the bottom green lines indicate $>2.0$ fold-change when comparing the expression of IncRNAs in the control and ALD-treated MC groups. (B) The number of up- and downregulated lncRNAs from the ALD-treated MCs and the control groups. (C) The numbers of upregulated and downregulated mRNAs from the ALD-treated MCs and the control groups. (D) Fold-change alterations for mRNAs expressed in the control and ALD-treated MC group. The number of points above the top and below the bottom green lines indicate $>2.0$ fold-change when comparing the expression of mRNAs in the control and ALD-treated MC groups ALD, aldosterone; lncRNAs, long non-coding RNAs; MCs, mesangial cells.

$R T$-qPCR analysis of microarray hybridization. To quantify the microarray hybridization results, RT-qPCR was performed on five upregulated lncRNAs (BC168211, BC088254, AF336872, AY325162 and BC168687), two downregulated lncRNAs (AF230638 and BC167085) (Fig. 2A), three upregulated mRNAs (NM_001108598, NM_001109190 and NM_001101018) (Fig. 2B) and three downregulated mRNAs (NM_019347, NM_177962 and NM_001108823) (Fig. 2C), selected on the basis of their levels of expression on the microarray and their biological significance. The RT-qPCR data was demonstrated to be consistent with the microarray results, with BC168211, BC088254, AF336872, AY325162 and BC168687 being upregulated and AF230638, BC167085 being downregulated compared with the control. The RT-qPCR data were again consistent with the microarray results for the mRNAs with NM_001108598, NM_001109190 and NM_001101018 being upregulated and NM_019347, NM_177962 and NM_001108823 being downregulated $(\mathrm{P}<0.05$ vs. the control group).

Expression signatures of differentially expressed lncRNAs. The general characteristics of the differentially expressed lncRNAs were summarized, including chromosomal, source, relationship and fold-change distribution. Chromosomal distribution of the number of up- or downregulated IncRNAs located on different chromosomes was demonstrated (Fig. 3A and B). Fold-change distribution demonstrated the differential expression of up- and downregulated lncRNAs (Fig. 3C and D), respectively. Source distribution respectively demonstrated the percentages of up- and downregulated lncRNAs collected from different sources (Fig. 4A and B), including misc_lncRNA, ultra-conserved region, Refseq-XR and mouse_ortholog. 

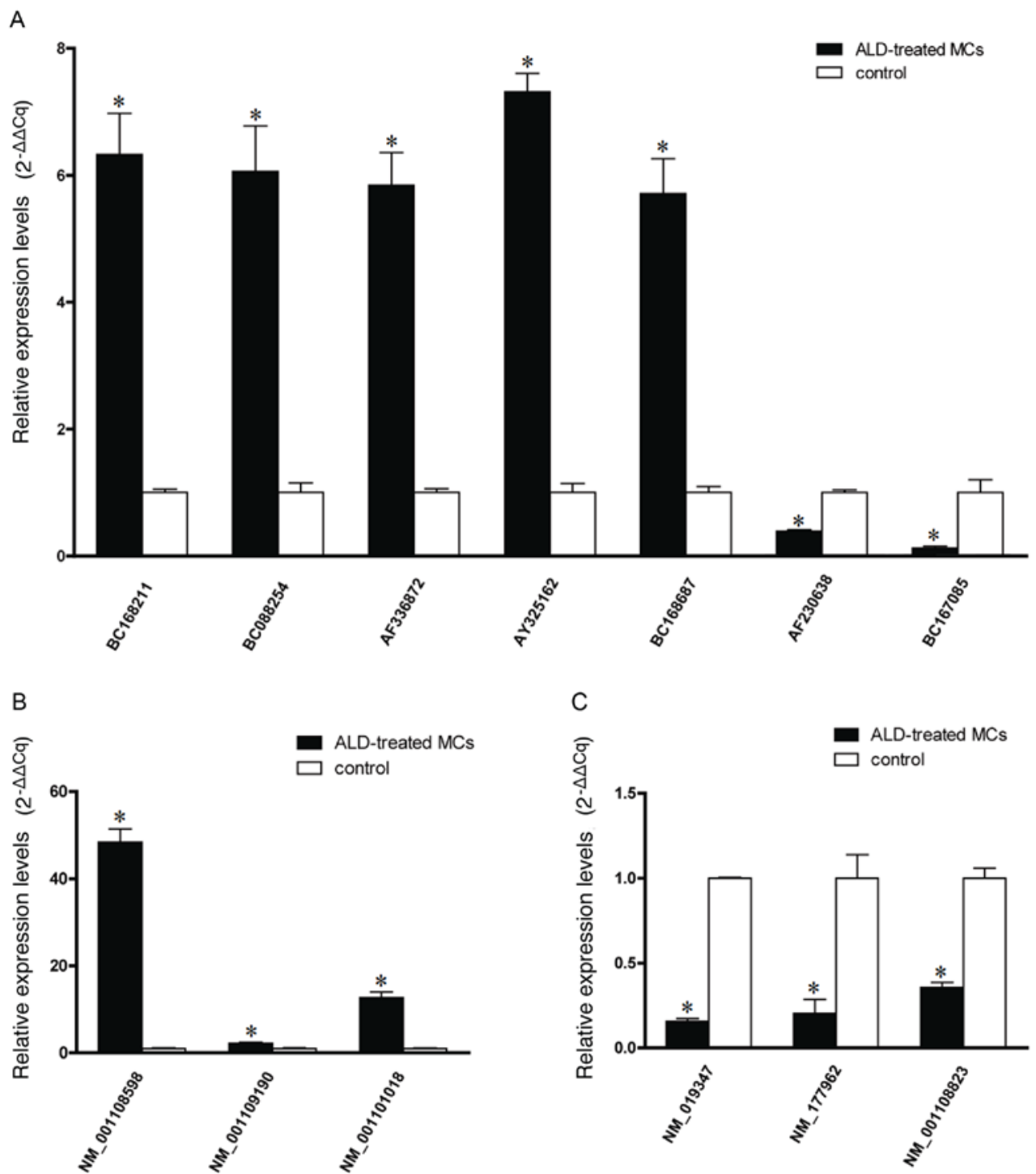

Figure 2. Reverse transcription-quantitative polymerase chain reaction quantification of microarray hybridization. (A) The relative expression level of lncRNAs BC168211, BC088254, AF336872, AY325162, BC168687, AF230638 and BC167085. (B) The relative expression levels of upregulated mRNAs, NM_001108598, NM_001109190 and NM_001101018, and (C) downregulated mRNAs, NM_019347, NM_177,962 and NM_0,011,08823. "P<0.05 vs. the control group. ALD, aldosterone; lncRNAs, long non-coding RNAs; MCs, mesangial cells.

Relationship distribution demonstrated the association of upand downregulated lncRNAs (Fig. 4C and D), respectively.

GOand pathwayanalysis. Toelucidate the biological processes and functional classification of differentially expressed lncRNAs, GO and pathway analyses were performed. The functions of coding genes adjacent to dysregulated lncRNAs included (in size order, most prevalent first, the top ten): i) Response to external stimuli; ii) response to stress; iii) positive regulation of biological processes; iv) cyclic nucleotide metabolic process; v) regulation of multicellular organism processes; vi) positive regulation of the apoptotic process; vii) cell division; viii) $\mathrm{M}$ phase; ix) mitosis; and $\mathrm{x}$ ) nuclear division (Fig. 5A). The cellular component containing dysregulated lncRNAs included the following (in size order, most prevalent first, the top ten): i) Cell periphery; ii) dendrite; iii) chloride channel complex; iv) neuron projection; v) extracellular region part; vi) hemoglobin complex; vii) extracellular space; viii) plasma membrane; ix) mitotic spindle; and $\mathrm{x}$ ) spindle
(Fig. 5B). The molecular function of dysregulated lncRNAs mainly consisted of the following (in size order, most prevalent first, the top ten): i) Ion channel activity; ii) potassium ion transmembrane transporter activity; iii) potassium channel activity; iv) transporter activity; v) passive transmembrane transporter activity; vi) ion transmembrane transporter activity; vii) protein binding; viii) oxygen transporter activity; ix) receptor binding; and $\mathrm{x}$ ) substrate-specific transmembrane transporter activity (Fig. 5C). Pathway analysis is a functional analysis process that maps genes to KEGG pathways. In the present study, the top 10 pathways that were associated with coding genes of dysregulated lncRNAs involved: i) Estrogen signaling pathway; ii) peroxisome proliferator-activated receptor (PPAR) signaling pathway; iii) cell cycle; iv) retrograde endocannabinoid signaling; v) circadian entrainment; vi) hypertrophic cardiomyopathy; vii) gastric acid secretion; viii) microRNAs in cancer; ix) cardiac muscle contraction; and $\mathrm{x}$ ) dilated cardiomyopathy-all Rattus norvegicus (Fig. 5D). 

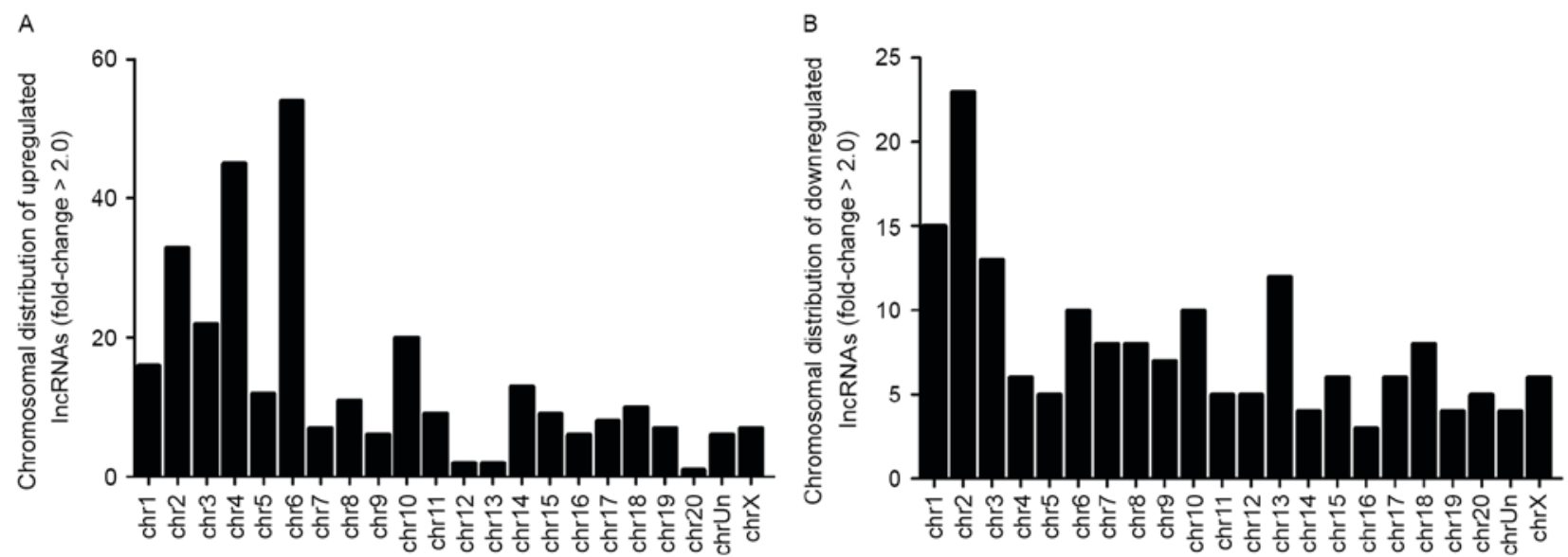

C

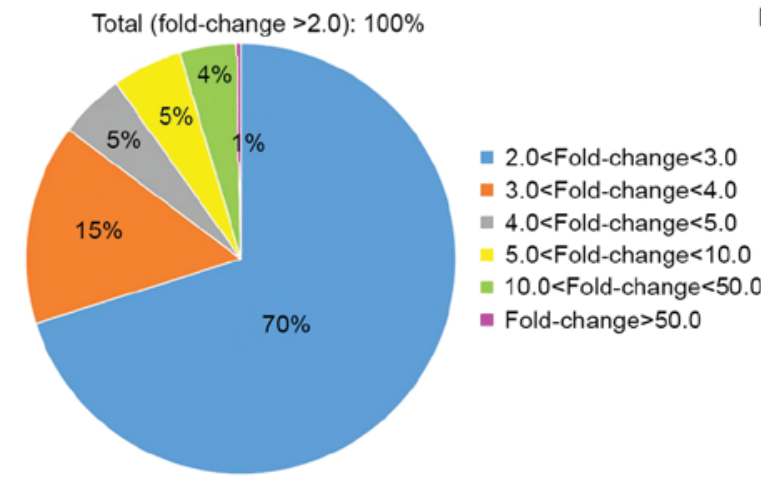

$\mathrm{D}$

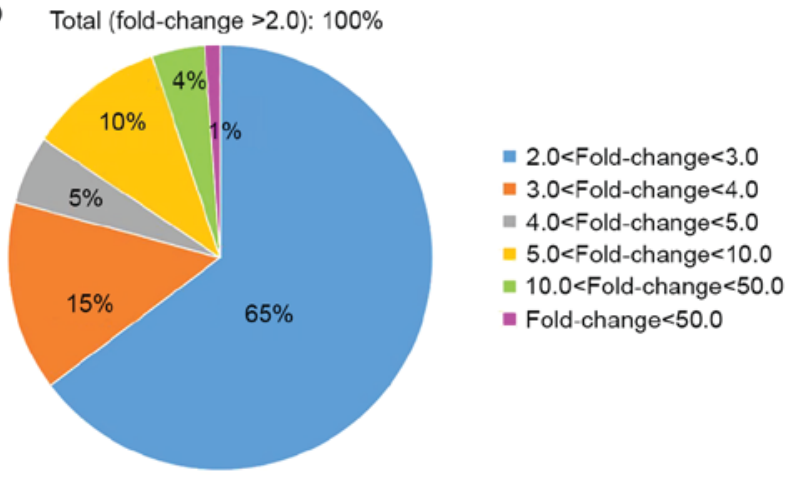

Figure 3. Chromosomal distribution demonstrating the number of (A) up- and (B) downregulated lncRNAs. Fold-change distribution demonstrating the differential expression of (C) up- and (D) downregulated lncRNAs. IncRNAs, long non-coding RNAs.

A

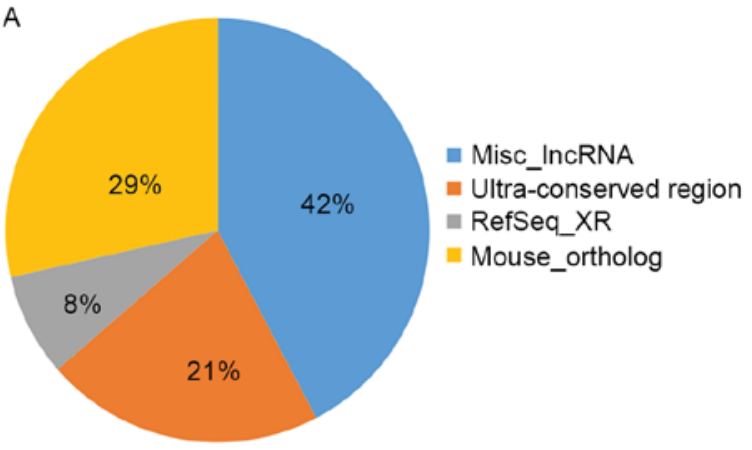

C

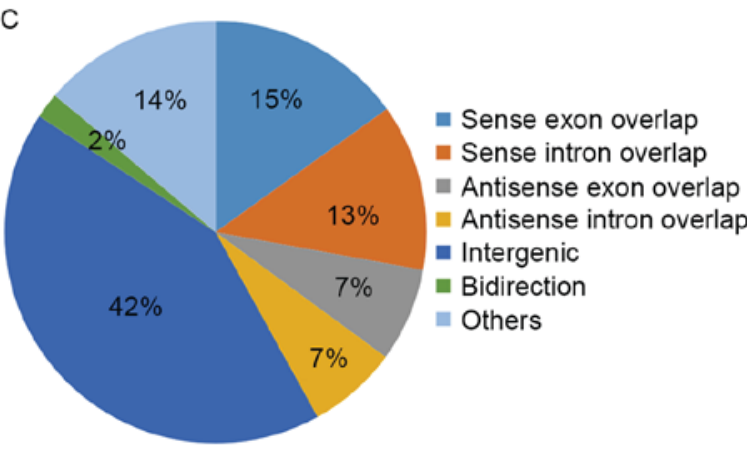

B

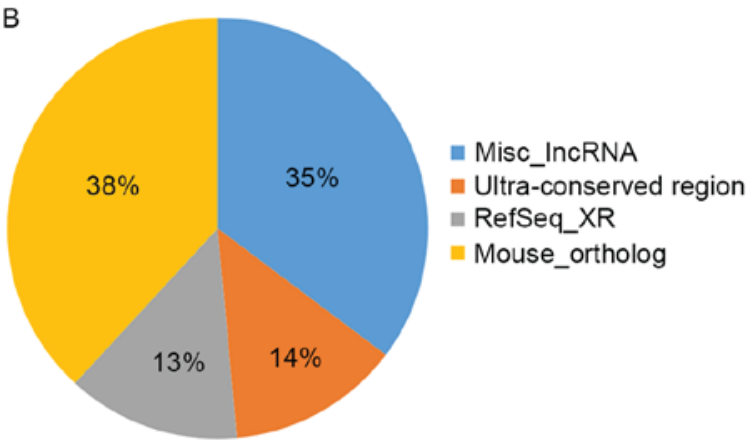

D

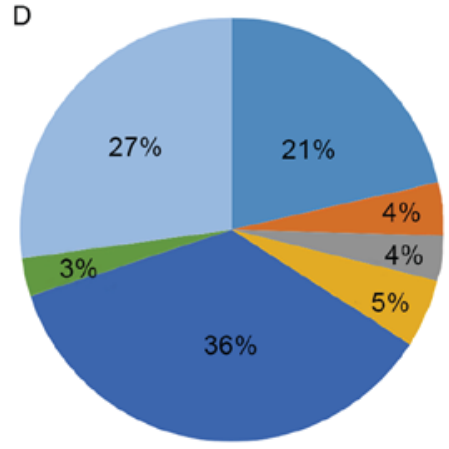

- Sense exon overlap - Sense intron overlap

antisense exon overlap

Antisense intron overlap

- Intergenic

- Bidirection

athers

Figure 4. Source distribution demonstrating the number of (A) up- and (B) downregulated lncRNAs as a percentage. Relationship distribution demonstrated the association of (C) up- and (D) downregulated lncRNAs. lncRNAs, long non-coding RNAs.

Bioinformatic analysis. As the transcription of non-coding genes can affect the expression of their flanking coding genes, relatively lowly and highly expressed lncRNAs were selected with a $<$ and $>3$-fold-change, respectively, as well as an raw 


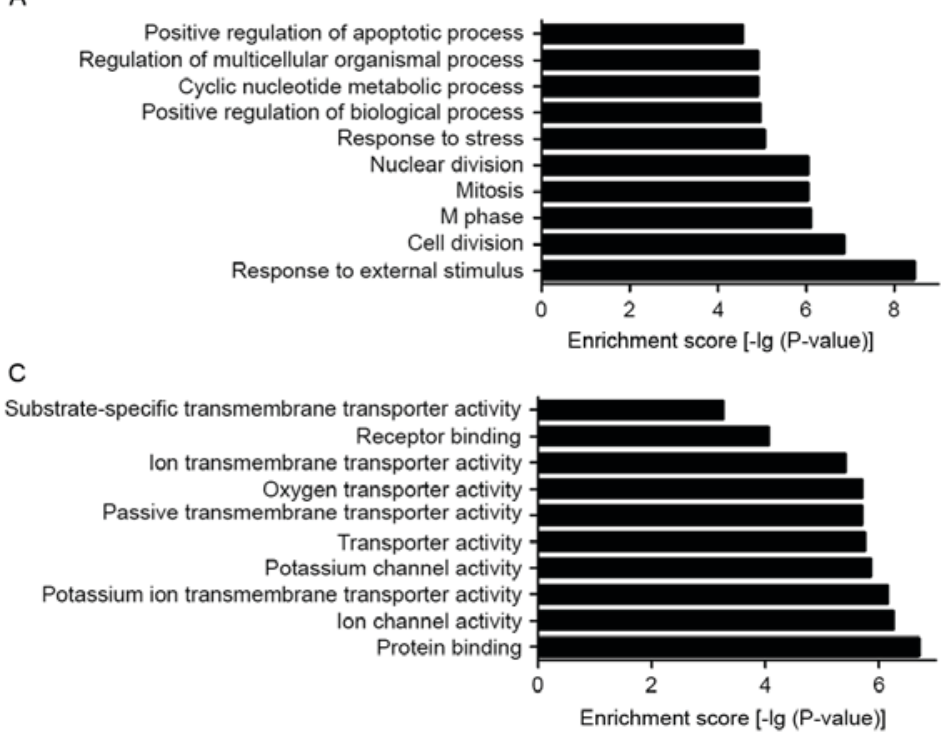

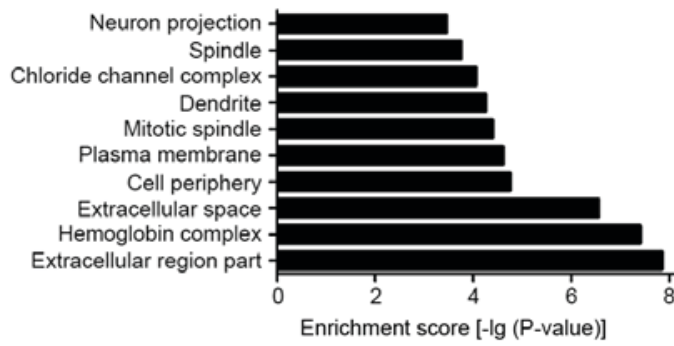

D

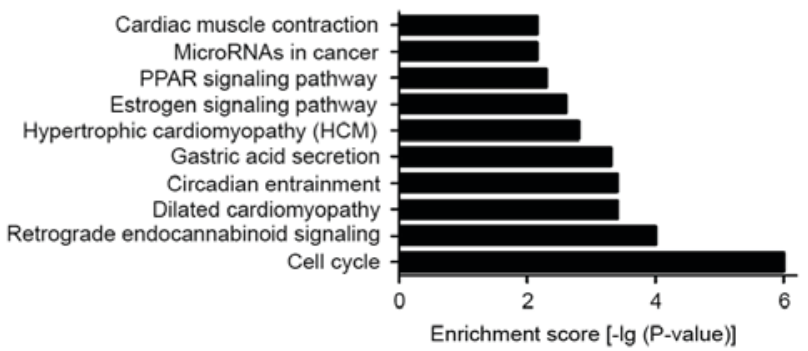

Figure 5. Biological process classification of GO analysis of (A) downregulated and up-regulated lncRNAs (counter-clockwise from the upper right to the upper left in accordance with size order from smallest to biggest), respectively. (B) Cellular component classification of GO analysis of down- and upregulated lncRNAs, respectively (the top 10 were selected, proportionately). (C) Molecular function classification of GO analysis of down- and upregulated lncRNAs, respectively (the top 10 were selected, proportionately). (D) The pathway of down- and up-regulated lncRNAs between stimulated and normal control groups, respectively (the top 10 were selected, proportionately). GO, gene ontology; lncRNAs, long non-coding RNAs.

$>100$ in the MCs treated with ALD and control group, and an associated coding gene with a function in a developmental processes, including cell cycle and PPAR signaling. It was demonstrated that dual specificity phosphatase 15 (Dusp15) and acyl-coenzyme A thioesterase Them4 (Them4) were associated with the lncRNAs BC168211 and BC168687, respectively (data not shown).

\section{Discussion}

Currently available therapies are not efficacious in the treatment of CKD, suggesting that further understanding of the molecular mechanisms underlying the pathogenesis of CKD is required for the identification of more effective diagnostic markers and therapeutic targets. MCs have been demonstrated to be a target of local ALD action, which may serve an important role in glomerular damage in CKD (36). In addition, ALD has been proved to serve a significant role in modulating MC function $(11,23,37)$.

The present study, to the best of the authors' knowledge, is the first to report the differential lncRNA expression in MCs treated with ALD compared with normal MCs. A threshold of $>3.0$ fold-change and raw $>100$ was set and it was demonstrated that 5 lncRNAs were upregulated and 2 were downregulated in MCs treated with ALD compared with the non-ALD treated MCs. The RT-qPCR results revealed that BC168211, BC088254, BC168687, AF336872 and AY325162 were significantly upregulated in MCs treated with ALD, and AF230638 and BC167085 were downregulated. Furthermore, it was demonstrated that lncRNAs may act through distinct transcription factors to modulate their target genes' transcription, thereby being involved in the potential mechanism of CKD. Then, mRNA microarray technology was used to evaluate differences in the mRNA expression profiles of control and MCs treated with ALD. GO and pathway analyses revealed that these IncRNAs were associated with changes in key pathogenic processes of CKD.

The collected data can be used to analyze the role of lncRNA transcripts in ALD-induced CKD. The GO project provides a controlled vocabulary that can be used to describe genes and gene product attributes (38). The biological processes involving dysregulated lncRNAs was demonstrated to be associated with cell proliferation stimulated by ALD included the following: Cell division; positive regulation of biological processes; response to external stimuli; regulation of multicellular organism processes; and cyclic nucleotide metabolic processes. The biological processes associated with lncRNAs included cell division, immune system processes, immune responses and cell-cell signaling. Pathway analysis provides a method for gaining insight into the underlying biology of differentially expressed genes and proteins (16). Pathway analysis demonstrated that the associated genes of dysregulated lncRNAs between the control and MCs treated with ALD included a variety of pathways for example cell cycle-associated, PPAR signaling and estrogen signaling pathways. It has been reported that ALD serves a major part in the glomerular ECM accumulation and proliferation of MCs in several glomerular diseases, and produces renal fibrosis in rats $(18-21,23,24)$. The 1 ncRNAs examined in the present study were demonstrated to be involved in the progression of CKD, which is induced by cell division, cell proliferation and immune deposits, in which ALD was involved.

Due to the complexity of the transcriptome, lncRNAs are frequently overlapping or are interspersed between multiple coding and non-coding transcripts $(39,40)$. In the present study, two genes were identified to be associated with lncRNAs through GO and pathway analysis. These results were used to investigate the association between lncRNAs and genes, further. Dusp15 and Them4 were demonstrated to be associated with the lncRNAs BC168211 and BC168687, respectively. 
Dusp15, a member of the protein tyrosine phosphatase family, previously only reported to be expressed in the testes, was suggested to be a pharmacological target for promoting remyelination in multiple sclerosis (41). Dusp15 is expressed in the kidneys of spontaneously hypertensive rats, which indicates that it is associated with renal injury. Dusp15 was identified to serve a role in the regulation of cell proliferation, positive regulation of the JNK cascade and TGF- $\beta$ receptor-signaling pathway. TGF- $\beta$ has been recognized to be an important factor in the development of CKD (42). In rat MCs, ALD upregulates mRNA expression of TGF- $\beta$ partly by enhancing the ERK1/2, JNK and AP-1 intracellular signaling pathways, and stimulating the progression of renal disease $(20,21,43-45)$. TGF- $\beta$ has also been demonstrated to induce mesangial expansion, which is caused by MC hypertrophy, proliferation and eventually apoptosis (46). In the present study, an association was demonstrated between BC168211 and Dusp15. The level of Dusp15 was revealed to be increased in stimulated MCs compared with the control cells. This may indicate that Dusp15 is involved in promoting the proliferation of MCs. However, the intrinsic association between BC168211 and Dusp15 is not completely understood. Further studies on this issue are planned.

Them4, a negative regulator of $\mathrm{RAC}-\alpha$ serine/threonine-protein kinase (Akt) and activated Akt, is known to protect the cell from apoptosis. The amino-terminal domain of Them4 may bind to Akt $(47,48)$. Human Them 4 has also been linked to Akt regulation and apoptosis (49). ALD stimulates MC proliferation via the phosphoinositide 3-kinase (PI3K)/Akt signaling pathway (50). The PI3K-Akt signaling pathway regulates fundamental cellular functions including transcription, translation, proliferation, growth and survival. PI3K catalyzes the production of phosphatidylinositol-3,4,5-triphosphate, which in turn serves as a second messenger that helps to activate Akt. Once active, Akt can control key cellular processes by phosphorylating substrates involved in apoptosis, protein synthesis, metabolism and cell cycle (50) (from KEGG source record: rno04151). Thus far, it has been clear that Them4 is associated with the cell cycle (49). In the present study it was hypothesized that Them 4 is involved in the PI3K/Akt signaling pathway in the progression of mesangial expansion. Them 4 was demonstrated to be upregulated in ALD stimulated MCs. However, the precise mechanism of Them 4 with BC168687 in ALD induced CKD warrants further investigation.

In conclusion, to the best of the author's knowledge, the present study is the first to provide a profile of lncRNAs in ALD-induced MCs in vitro. A network of differentially expressed lncRNAs was constructed, and numerous IncRNAs are involved in the development and mechanism of CKD. Further investigation of the biological progresses and molecular mechanisms of the dysregulated lncRNAs is necessary. The present study may provide novel insights into the molecular basis of CKD, and aid the identification of potential novel biomarkers and development of therapeutic interventions for this disease.

\section{Acknowledgements}

The present study was sponsored by the Scientific Research Program of Nanjing Medical University (grant no. 2015NJMUZD028) and the Natural Science Fund Project of Colleges in Jiangsu Province, China (grant no. 15KJD320005).

\section{References}

1. Zhang L, Zhang P, Wang F, Zuo L, Zhou Y, Shi Y, Li G, Jiao S, Liu Z, Liang W and Wang H: Prevalence and factors associated with CKD: A population study from Beijing. Am J Kidney Dis 51: 373-384, 2008.

2. Zeisberg M, Khurana M, Rao VH, Cosgrove D, Rougier JP, Werner MC, Shield CF III, Werb Z and Kalluri R: Stage-specific action of matrix metalloproteinases influences progressive hereditary kidney disease. PLoS Med 3: e100, 2006.

3. Jo YI, Cheng H, Wang S, Moeckel GW and Harris RC: Puromycin induces reversible proteinuric injury in transgenic mice expressing cyclooxygenase-2 in podocytes. Nep Exp Nephrol 107: e87-e94, 2007.

4. Pesce CM, Striker LJ, Peten E, Elliot SJ and Striker GE: Glomerulosclerosis at both early and late stages is associated with increased cell turnover in mice transgenic for growth hormone. Lab Invest 65: 601-605, 1991.

5. Kreisberg JI and Karnovsky MJ: Glomerular cells in culture. Kidney Int 23: 439-447, 1983.

6. Floege J, Burns MW, Alpers CE, Yoshimura A, Pritzl P, Gordon K, Seifert RA, Bowen-Pope DF, Couser WG and Johnson RJ: Glomerular cell proliferation and PDGF expression precede glomerulosclerosis in the remnant kidney model. Kidney Int 41: 297-309, 1992.

7. Schlöndorff D and Banas B: The mesangial cell revisited: No cell is an island. J Am Soc Nephrol 20: 1179-1187, 2009.

8. Eddy AA and Neilson EG: Chronic kidney disease progression. J Am Soc Nephrol 17: 2964-2966, 2006.

9. Siragy HM and Xue C: Local renal aldosterone production induces inflammation and matrix formation in kidneys of diabetic rats. Exp Physiol 93: 817-824, 2008.

10. Wu P, Liang X, Dai Y, Liu H, Zang Y, Guo Z, Zhang R, Lai W, Zhang Y and Liu Y: Aldosterone biosynthesis in extraadrenal tissues. Chin Med J (Engl) 112: 414-418, 1999.

11. Nishikawa T, Suematsu S, Saito J, Soyama A, Ito H, Kino T and Chrousos G: Human renal mesangial cells produce aldosterone in response to low-density lipoprotein (LDL). J Steroid Biochem Mol Biol 96: 309-316, 2005.

12. Martinez D, Oestreicher E, Roubsanthisuk W, et al: Angiotensin II, aldosterone and the caveolae. IV. Aldosterone content of the kidney after adrenalectomy; proceedings of the Journal of Hypertension, Lippincott Williams \& Wilkins, Philadelphia, PA, 19106-3621, 2002.

13. Silvestre JS, Heymes C, Oubénaïssa A, Robert V, Aupetit-Faisant B, Carayon A, Swynghedauw B and Delcayre C: Activation of cardiac aldosterone production in rat myocardial infarction: effect of angiotensin II receptor blockade and role in cardiac fibrosis. Circulation 99: 2694-2701, 1999.

14. Silvestre JS, Robert V, Heymes C, Aupetit-Faisant B, Mouas C, Moalic JM, Swynghedauw B and Delcayre C: Myocardial production of aldosterone and corticosterone in the rat. Physiological regulation. J Biol Chem 273: 4883-4891, 1998.

15. Kayes-Wandover KM and White PC: Steroidogenic enzyme gene expression in the human heart. J Clin Endocrinol Metab 85: 2519-2525, 2000.

16. Takeda Y, Yoneda T, Demura M, Miyamori I and Mabuchi H: Sodium-induced cardiac aldosterone synthesis causes cardiac hypertrophy. Endocrinology 141: 1901-1904, 2000.

17. Kornel L: Colocalization of 11 beta-hydroxysteroid dehydrogenase and mineralocorticoid receptors in cultured vascular smooth muscle cells. Am J Hypertens 7: 100-103, 1994.

18. Lai LY, Gu Y, Chen J, Yu SQ, Ma J, Yang HC and Lin SY: Production of aldosterone by rat mesangial cell and the accumulation of extracellular matrix induced by aldosterone. Zhonghua Yi Xue Za Zhi 83: 1900-1905, 2003 (In Chinese).

19. Terada Y, Kuwana H, Kobayashi T, Okado T, Suzuki N, Yoshimoto T, Hirata Y and Sasaki S: Aldosterone-stimulated SGK1 activity mediates profibrotic signaling in the mesangium. J Am Soc Nephrol 19: 298-309, 2008.

20. Han JS, Choi BS, Yang CW and Kim YS: Aldosterone-induced TGF-betal expression is regulated by mitogen-activated protein kinases and activator protein-1 in mesangial cells. J Korean Med Sci 24 (Suppl 1): S195-S203, 2009. 
21. Li XD, Chen XW, Tang DS, Liang D and Liu HF: Effects of aldosterone on synthesis of fibronectin and expression of transforming growth factor-beta $1 \mathrm{mRNA}$ in cultured rat mesangial cells. Xi Bao Yu Fen Zi Mian Yi Xue Za Zhi 23: 140-1422, 2007 (In Chinese).

22. Terada Y, Ueda S, Hamada K, Shimamura Y, Ogata K, Inoue K, Taniguchi Y, Kagawa T, Horino T and Takao T: Aldosterone stimulates nuclear factor-kappa B activity and transcription of intercellular adhesion molecule-1 and connective tissue growth factor in rat mesangial cells via serum-and glucocorticoid-inducible protein kinase-1. Clin Exp Nephrol 16: 81-88, 2012.

23. Terada Y, Kobayashi T, Kuwana H, Tanaka H, Inoshita S, Kuwahara M and Sasaki S: Aldosterone stimulates proliferation of mesangial cells by activating mitogen-activated protein kinase 1/2, cyclin D1, and cyclin A. J Am Soc Nephrol 16 : 2296-2305, 2005.

24. Yuan J, Jia R and Bao Y: Aldosterone up-regulates production of plasminogen activator inhibitor-1 by renal mesangial cells. J Biochem Mol Biol 40: 180-188, 2007.

25. Wang KC and Chang HY: Molecular mechanisms of long noncoding RNAs. Mol Cell 43: 904-914, 2011.

26. Ponting CP, Oliver PL and Reik W: Evolution and functions of long noncoding RNAs. Cell 136: 629-641, 2009.

27. Cai B, Song XQ, Cai JP and Zhang S: HOTAIR: A cancer-related long non-coding RNA. Neoplasma 61: 379-391, 2014.

28. Mercer TR, Dinger ME and Mattick JS: Long non-coding RNAs: Insights into functions. Nat Rev Genet 10: 155-159, 2009.

29. Zhang A, Han Y, Wang B, Li S and Gan W: Beyond gap junction channel function: The expression of $\mathrm{Cx} 43$ contributes to aldosterone-induced mesangial cell proliferation via the ERK1/2 and PKC pathways. Cell Physiol Biochem 36: 1210-1222, 2015.

30. Xu G, Chen J, Pan Q, Huang K, Pan J, Zhang W, Chen J, Yu F, Zhou $\mathrm{T}$ and Wang Y: Long noncoding RNA expression profiles of lung adenocarcinoma ascertained by microarray analysis. PLoS One 9: e104044, 2014.

31. Khalil AM, Guttman M, Huarte M, Garber M, Raj A, Rivea Morales D, Thomas K, Presser A, Bernstein BE, van Oudenaarden A, et al: Many human large intergenic noncoding RNAs associate with chromatin-modifying complexes and affect gene expression. Proc Natl Acad Sci USA 106: 11667-11672, 2009.

32. Guttman M, Amit I, Garber M, French C, Lin MF, Feldser D, Huarte M, Zuk O, Carey BW, Cassady JP, et al: Chromatin signature reveals over a thousand highly conserved large non-coding RNAs in mammals. Nature 458: 223-227, 2009.

33. Yang Y, Li H, Hou S, Hu B, Liu J and Wang J: The noncoding RNA expression profile and the effect of lncRNA AK126698 on cisplatin resistance in non-small-cell lung cancer cell. PLoS One 8: e65309, 2013.

34. Livak KJ and Schmittgen TD: Analysis of relative gene expression data using real-time quantitative PCR and the 2(-Delta Delta C(T)) method. Methods 25: 402-408, 2001.

35. Ashburner M, Ball CA, Blake JA, Botstein D, Butler H, Cherry JM, Davis AP, Dolinski K, Dwight SS, Eppig JT, et al: Gene ontology: Tool for the unification of biology. The Gene Ontology Consortium. Nat Genet 25: 25-29, 2000.

36. Lai L, Chen J, Hao CM, Lin S and Gu Y: Aldosterone promotes fibronectin production through a Smad2-dependent TGF-beta1 pathway in mesangial cells. Biochem Biophys Res Commun 348 : $70-75,2006$
37. Nishiyama A, Yao L, Nagai Y,Miyata K, Yoshizumi M,Kagami S, Kondo S, Kiyomoto H, Shokoji T, Kimura S, et al: Possible contributions of reactive oxygen species and mitogen-activated protein kinase to renal injury in aldosterone/salt-induced hypertensive rats. Hypertension 43: 841-448, 2004

38. Balakrishnan R, Harris MA, Huntley R, Van Auken K and Cherry JM: A guide to best practices for Gene Ontology (GO) manual annotation. Database (Oxford) 2013: bat054, 2013.

39. Carninci P, Kasukawa T, Katayama S, Gough J, Frith MC, Maeda N, Oyama R, Ravasi T, Lenhard B, Wells C, et al: The transcriptional landscape of the mammalian genome. Science 309: 1559-1163, 2005.

40. Kapranov P, Drenkow J, Cheng J, Long J, Helt G, Dike S and Gingeras TR: Examples of the complex architecture of the human transcriptome revealed by RACE and high-density tiling arrays. Genome Res 15: 987-997, 2005.

41. Schmidt F, van den Eijnden M, Pescini Gobert R, Saborio GP, Carboni S, Alliod C, Pouly S, Staugaitis SM, Dutta R, Trapp B, et al: Identification of $\mathrm{VHY} / \mathrm{Dusp15}$ as a regulator of oligodendrocyte differentiation through a systematic genomics approach. PLoS One 7: e40457, 2012.

42. Schnaper HW, Jandeska S, Runyan CE, Hubchak SC, Basu RK, Curley JF, Smith RD and Hayashida T: TGF-beta signal transduction in chronic kidney disease. Front Biosci (Landmark Ed) 14: 2448-2465, 2009.

43. Chin BY, Mohsenin A,Li SX, Choi AM and Choi ME: Stimulation of pro-alpha(1)(I) collagen by TGF-beta(1) in mesangial cells: role of the p38 MAPK pathway. Am J Physiol Renal Physiol 280: F495-F504, 2001.

44. Huwiler A and Pfeilschifter J: Transforming growth factor beta 2 stimulates acute and chronic activation of the mitogen-activated protein kinase cascade in rat renal mesangial cells. FEBS Lett 354: 255-258, 1994.

45. Martínez-Salgado C, Rodríguez-Peña AB and López-Novoa JM: Involvement of small Ras GTPases and their effectors in chronic renal disease. Cell Mol Life Sci 65: 477-492, 2008.

46. López-Hernández FJ and López-Novoa JM: Role of TGF- $\beta$ in chronic kidney disease: An integration of tubular, glomerular and vascular effects. Cell Tissue Res 347: 141-154, 2012.

47. Cao J, Xu H, Zhao H, Gong W and Dunaway-Mariano D: The mechanisms of human hotdog-fold thioesterase 2 (hTHEM2) substrate recognition and catalysis illuminated by a structure and function based analysis. Biochemistry 48: 1293-1304, 2009.

48. Zhao H, Martin BM, Bisoffi M and Dunaway-Mariano D: The Akt C-terminal modulator protein is an acyl-CoA thioesterase of the Hotdog-Fold family. Biochemistry 48: 5507-5509, 2009.

49. Zhao H,Lim K, Choudry A, Latham JA, Pathak MC, Dominguez D, Luo L, Herzberg O and Dunaway-Mariano D: Correlation of structure and function in the human hotdog-fold enzyme hTHEM4. Biochemistry 51: 6490-6492, 2012.

50. Nishiyama A, Yao L, Fan Y, Kyaw M, Kataoka N, Hashimoto K, Nagai Y, Nakamura E, Yoshizumi M, Shokoji T, et al: Involvement of aldosterone and mineralocorticoid receptors in rat mesangial cell proliferation and deformability. Hypertension 45: 710-716, 2005 . 